\title{
The Role of Multiple Contralesional Motor Areas for Complex Hand Movements after Internal Capsular Lesion
}

\author{
Martin Lotze, ${ }^{1,2}$ Jochen Markert, ${ }^{1}$ Paul Sauseng, ${ }^{3}$ Julia Hoppe, ${ }^{1,4}$ Christian Plewnia, ${ }^{5}$ and Christian Gerloff ${ }^{1,4}$ \\ ${ }^{1}$ Department of General Neurology, Hertie Institute for Clinical Brain Research, University of Tuebingen, D-72076 Tuebingen, Germany, ${ }^{2}$ Institute of \\ Medical Psychology and Behavioral Neurobiology, University of Tuebingen, D-72074 Tuebingen, Germany, ${ }^{3}$ Department of Psychology, University of \\ Salzburg, A-5020 Salzburg, Austria, ${ }^{4}$ Department of Neurology, University Medical Center Hamburg-Eppendorf, D-20246 Hamburg, Germany, and \\ ${ }^{5}$ Department of Psychiatry and Psychotherapy, University of Tuebingen, D-72072 Tuebingen, Germany
}

Imaging techniques document enhanced activity in multiple motor areas of the damaged and contralesional (intact) hemisphere $(\mathrm{CON}-\mathrm{H})$ after stroke. In the subacute stage, increased activity within motor areas in the CON-H during simple movements of the affected hand has been shown to correlate with poorer motor outcome. For those patients in the chronic stage who recovered well, the functional relevance of an increased activation within the $\mathrm{CON}-\mathrm{H}$ is unclear. Using trains of repetitive transcranial magnetic stimulation (TMS) during performance of complex finger movements, we tested the behavioral relevance of regional functional magnetic resonance imaging (fMRI) activation within the $\mathrm{CON}-\mathrm{H}$ for sequential finger movement performance of the recovered hand in seven patients who had experienced a subcortical stroke. TMS was navigated over fMRI activation maxima within anatomically preselected regions of the $\mathrm{CON}-\mathrm{H}$, and effects were compared with those of healthy controls. Stimulation over the dorsal premotor cortex (dPMC), the primary motor cortex (M1), and the superior parietal lobe (SPL) resulted in significant interference with recovered performance in patients. Interference with the dPMC and M1 induced timing errors only, SPL stimulation caused both timing and accuracy deficits. The present results argue for a persistent beneficial role of the $\mathrm{APMC}, \mathrm{M1}$, and SPL of the $\mathrm{CON}-\mathrm{H}$ on some aspects of effectively recovered complex motor behavior after subcortical stroke.

Key words: motor cortex; stroke; navigation; fMRI; plasticity; motor control

\section{Introduction}

Motor impairment after stroke recovers to varying degrees over time (Kotila et al., 1984). In monkeys, retraining skilled hand use after lesions of the cortical hand area results in expansion of the hand representation into regions formerly controlling the elbow and shoulder (Nudo et al., 1996). Also in humans, motor-related areas in the damaged hemisphere (DAM-H) that have not been destroyed or disconnected by the infarction are relevant for recovery (Chollet et al., 1991; Seitz et al., 1998). Less clear is the relevance of the contralesional hemisphere $(\mathrm{CON}-\mathrm{H})$, but there is evidence that both the DAM-H and the CON-H show substantial upregulation of activity in patients when compared with healthy controls (HCs) (Ward et al., 2003; Gerloff et al., 2006).

Beyond regional activation, interhemispheric interactions have been shown to contribute to unilateral motor performance in healthy subjects (Kobayashi et al., 2004) but also in stroke patients (Murase et al., 2004; Duque et al., 2005). In these patients, interhemispheric inhibition from the CON-H to the DAM-H is pathologically enhanced (Murase et al., 2004; Duque et al., 2005).

Received 0ct. 25, 2005; revised April 18, 2006; accepted April 18, 2006.

This work was supported by Deutsche Forschungsgemeinschaft Grants DFG SFB 550/TP C5 and DFG TH 812/1-1.

Correspondence should be addressed to Dr. Christian Gerloff, Department of Neurology, University Medical Center Ham-

burg-Eppendorf, Martinistrasse 52, D-20246 Hamburg, Germany. E-mail: gerloff@uke.uni-hamburg.de.

DOI:10.1523/JNEUROSCI.4564-05.2006

Copyright $\odot 2006$ Society for Neuroscience $\quad 0270-6474 / 06 / 266096-07 \$ 15.00 / 0$
A model to understand mechanisms of motor recovery is focal ischemic lesions of the pyramidal tract. These patients sometimes show rapid recovery of motor function (Fries et al., 1993), and imaging techniques have documented enhanced activity in multiple motor areas of the DAM-H and CON-H (Weiller et al., 1992; Gerloff et al., 2006), including secondary motor areas known to project to the spinal cord (Maier et al., 2002). However, the behavioral relevance of these activity increases has been questioned (Ward et al., 2003).

The behavioral relevance of regional activation can be tested by temporary interference with activation in target areas by transcranial magnetic stimulation (TMS) and subsequent analysis of induced behavioral deficits (Gerloff et al., 1998). This approach to interfere with behavioral function has also been referred to as "jamming." Studies addressing the functional relevance of areas with increased functional magnetic resonance imaging (fMRI)based activity in stroke patients are scarce. Single-pulse TMS over the primary motor cortex (M1) or the dorsal premotor cortex (dPMC) of the DAM-H results in increased reaction times (Werhahn et al., 2003; Fridmann et al., 2004). With respect to the CON-H, single-pulse TMS during simple reaction-time tasks points to a role of the dPMC (Johansen-Berg et al., 2002a), particularly in patients with severe motor deficits (Johansen-Berg et al., 2002b).

An increased contribution of motor areas within the CON-H can be expected particularly during complex finger movements 


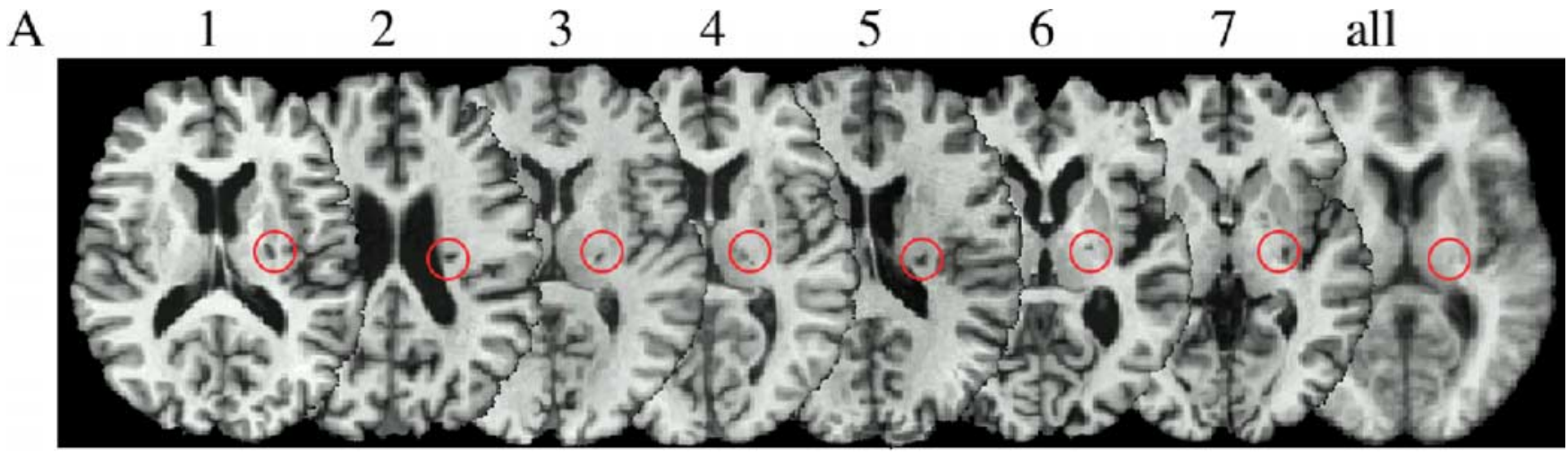

B

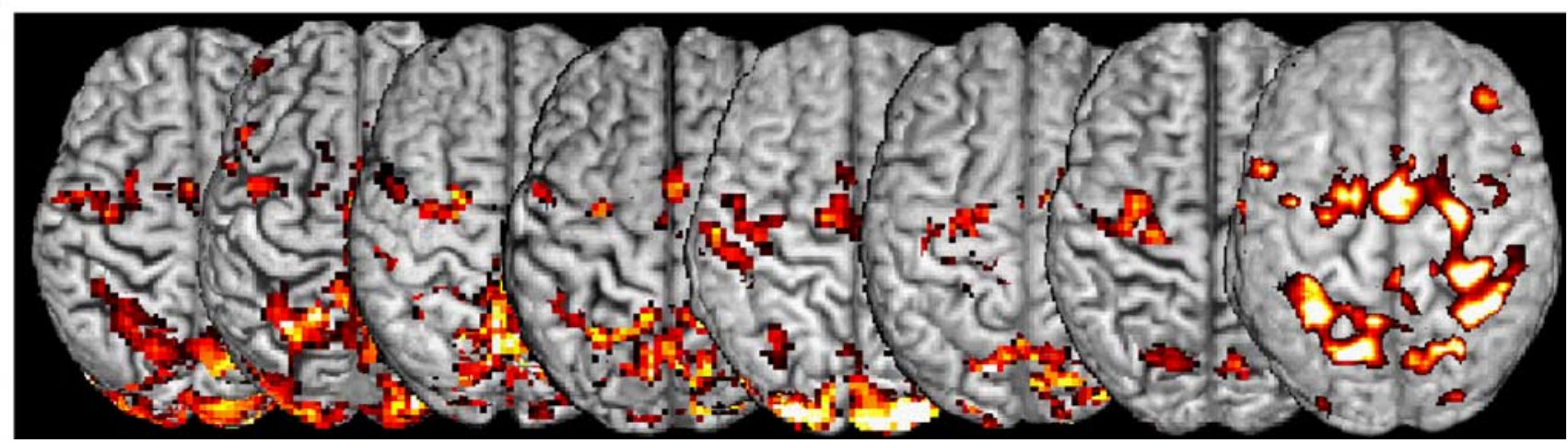

Figure 1. A, Subcortical lesions of the patients all flipped to the right side and are marked with a red cycle. The average T1 image (right) shows the lesion in the posterior internal capsule at group level. $\boldsymbol{B}$, fMRI results for each patient during performing the finger sequence with their formerly impaired hand; only the CON-H is shown for each patient. The right activation map (smoothed for overlay with $6 \mathrm{~mm}$ ) shows the group effect of all patients for the $\mathrm{CON}-\mathrm{H}$ (left) and the DAM-H (right) projected on the segmented T1 anatomy Montreal Neurological Institute reference brain. Note the interindividual variability of activation maxima within the precentral gyrus.

(Foltys et al., 2003). Therefore, we hypothesized that additional areas of the $\mathrm{CON}-\mathrm{H}$, beyond the $\mathrm{dPMC}$, gain behavioral relevance for recovered hand motor control when more challenging motor acts are required. To test this, we applied TMS jamming to motor-related regions of the CON-H in stroke patients executing complex finger movements with their recovered hand. All patients were in the chronic phase and had experienced near complete motor recovery. TMS was stereotactically navigated based on individual three-dimensional reconstructed fMRI-activation maps. The effects of TMS interference on motor behavior were compared in patients and age-matched HC subjects for timing errors and accuracy of performance.

\section{Materials and Methods}

Subjects. We investigated seven patients [six males; average age, $63.7 \pm$ (SD) 8.6 years; range, 51-76 years; all right-handed as tested according to Oldfield (1971)] with first-ever ischemic stroke in the internal capsule $>8$ months ago (average, $33.9 \pm 20.8$ ) (Fig. 1). All patients were severely paretic or even hemiplegic at their first day after stroke, four in their left hand and three in their right hand [initial median Medical Research Council (MRC) (Daniels and Worthingham, 1972) scale of the forearm muscle strength of 1] (see supplemental Table 1, available at www. jneurosci.org as supplemental material). All patients experienced almost complete recovery of motor function, leaving them with a median $\mathrm{Na}$ tional Institutes of Health stroke scale status (Lyden et al., 1994) of 1 and an MRC scale of 5 (no difference to the nonaffected side). Additionally, seven age-matched HCs [two males; average age, $63.9 \pm 4.5$ years; range, 56-69; all strongly right-handed as tested after Oldfield (1971)] who had no neurological or psychiatric illness were investigated with the same method.

Both groups gave their written informed consent to the experiment, which was approved by the University of Tuebingen ethics committee.
Motor task during scanning. Both patients and HCs performed a sequential finger-tapping task of 10 visually presented numbers indicating button presses with digits 2, 3, 4, and 5 (corresponding to the index, middle, ring, and small finger, respectively). All subjects trained tapping with maximum tapping velocity (without making mistakes in sequence order) for $10 \mathrm{~min}$ for each hand before fMRI measurement. Additionally, all patients trained and performed the sequence paced by a metronome $(1 \mathrm{~Hz})$. Patients had to perform tapping with their recovered hand (both maximal frequency, $2.0 \pm 0.6 \mathrm{~Hz}$ and $1 \mathrm{~Hz}$ ), and $\mathrm{HCs}$ had to perform tapping with their left hand $(2.4 \pm 0.6 \mathrm{~Hz}$; right vs left; NS). The $1 \mathrm{~Hz}$ frequency preceded the maximum-velocity finger sequence. Both were displayed by a video projector via a mirror fixated on the head coil. Conditions were performed in a block design alternating rest and performance four times on specially constructed MR-suitable keyboards with physiologically ordered buttons of $1.5 \mathrm{~cm}$ in diameter, connected via an optic-fiber cable to a recording computer outside of the scanner room, and recorded with Labview (version 5.6; National Instruments, Austin, TX).

fMRI scanning parameters. Subjects underwent MRI scanning at $3 \mathrm{~T}$ (Trio, 8 high-frequency head coil; Siemens, Erlangen, Germany) with 30 oblique transverse slices ( $3 \mathrm{~mm}$ thickness, $1 \mathrm{~mm}$ gap) covering the entire head using a T2*-weighted echo-planar imaging (EPI) sequence [repetition time (TR), $2 \mathrm{~s}$; matrix size, $64 \times 64$; echo time (TE), $30 \mathrm{~ms}$; flip angle, $90^{\circ}$. The subjects were in a supine position on the padded scanner couch and wore hearing protection. Additionally, a T1-weighted threedimensional image (MPrage; TR, $2.3 \mathrm{~s}$; TE, $3.93 \mathrm{~ms} ; 160$ sagittal slices $1+$ $0.5 \mathrm{~mm}$ ) was acquired.

Imaging analyses. Spatial preprocessing and data analysis were performed with SPM2 (Wellcome Department of Neuroscience, London, UK). Each time series was realigned and resliced after unwarping in a phase encoding direction (anterior/posterior) to account for movement and susceptibility artifacts. Before coregistration of EPI sequences to the T1-weighted anatomical image, it was reduced in spatial resolution to 
Table 1. Group results: patients minus HCs

\begin{tabular}{lllll}
\hline Area activated & $t$ value & $z$ value & $p_{\text {corr }}{ }^{a}$ & MNI coordinates \\
\hline idPMC & 7.46 & 4.48 & 0.01 & $-27,18,57$ \\
cdPMC & 5.15 & 3.67 & 0.04 & $24,3,63$ \\
iSPL & 4.65 & 3.45 & 0.04 & $-18,-54,60$ \\
CSPL & 4.22 & 3.24 & 0.04 & $15,-51,66$ \\
cM1 & 3.97 & 3.11 & 0.04 & $18,-30,66$ \\
iM1 & 3.26 & 2.70 & 0.05 & $-42,2,54$ \\
\hline
\end{tabular}

i, I psilateral to the hand moved; $c$, contralateral to the hand moved; MNI, Montreal Neurological Institute. ${ }^{a} p$ value corrected for false discovery rate ROls.

that of the EPI sequences $(3 \times 3 \times 3 \mathrm{~mm})$. For group comparison, data were smoothed with a Gaussian filter of $9 \mathrm{~mm}$ (full width at half maximum). For data used for TMS navigation, a filter size of $4 \mathrm{~mm}$ was used to achieve a high precision of navigation within functional maps. Data were high-pass filtered (128 s) and statistically evaluated for each individual. Conditions were modeled with a canonical hemodynamic response function using standard SPM2 settings. For navigation, significance thresholds between $<0.05$ and $<0.001$, corrected for multiple comparisons (FWE), were individually adjusted to detect activated voxels within the regions of interest (ROIs) in the $\mathrm{CON}-\mathrm{H}$ or left hemisphere of HCs, respectively. Functional images were saved and underwent further superposition with the T1 anatomy and segmentation by using Brainsight frameless software (Rogue Research, Montreal, Quebec, Canada). fMRI group results for the patients (Fig. 1, right) were normalized to the SPM template brain after segmentation to increase normalization preciseness. Random-effects $t$ statistics across subjects were calculated separately for each condition ( $p<0.005$; FWE corrected). Differences between patients and controls were compared with the two-sample random-effect $t$ test for all patients against all controls (Table 1) using a false discovery rate correction of $p<0.05$ for the selected ROIs. All regions were detected with the Automated Anatomical Labeling software (Tzourio-Mazoyer et al., 2002). For visualization in Figure 1, patients with right-sided paresis were radiologically normalized to achieve a constant representation of the intact hemisphere on the left side. T1weighted anatomical images were brain extracted (Steve Smith; http:// www.fmrib.ox.ac.uk/fsl) by using MRIcro software (http:// www.sph.s.c.edu/comd/rorden/mricro.html). Activation maps were superimposed on these extracted brains in a three-dimensional top view.

TMS parameters and resting motor threshold. At least 1 week after fMRI measurement, subjects were investigated with TMS using a Magstim rapid stimulator (Magstim, Whitland, UK) with a maximum output of 2.2 tesla equipped with a figure-eight coil, each wing with a diameter of $7 \mathrm{~cm}$.

TMS was delivered with the coil held tangentially to the scalp, $45^{\circ}$ from the mid-sagittal line, with the handle pointing backward. The current induced in the brain was therefore directed approximately perpendicular to the line of the central sulcus (Werhahn, 1994). For sham stimulation, the coil was turned $90^{\circ}$ so that only one wing touched the scalp at the $\mathrm{Cz}$ position (10/20 system), but the pulses were not applied to the head surface.

The resting motor threshold, measured for the hand flexors of the nonperforming intact hand (respectively, the right hand in controls) was defined as the minimum stimulus intensity that produced motor-evoked potentials of at least $50 \mu \mathrm{V}$ peak-to-peak amplitude in at least thee of five trials (Rossini et al., 1994).

Neuronavigation. For navigation of target regions, we used a frameless, fMRI-guided stereotaxic system with a Polaris IR tracker camera (Northern Digital, Waterloo, Ontario, Canada) and Brainsight frameless software (Rogue, Montreal, Quebec, Canada). fMRI maps were overlayed on the T1-weighted anatomy, and the brain was manually segmented and visualized for the three-dimensional 3-6 $\mathrm{mm}$ surface rendering. ROIs were selected before the beginning of the study. We tested three different verum locations (Fig. 2): the dPMC [located as the fMRI-activation maximum anterior to the precentral sulcus, inferior to the superior frontal sulcus at the posterior part of the medial frontal sulcus (Fridman et al., 2004)], M1 [posterior half of the precentral gyrus; location of activation maxima nearest to the anatomical hand knob (Yousry et al., 1997)], and the superior parietal lobe (SPL; posterior to the postcentral sulcus and superior to the parietal sulcus). The TMS coil was navigated to the individual fMRI-activation maxima within the preselected ROIs. No further adjustments were made to elicit maximal motor responses after stimulation of CON-H M1. Additionally, we tested three different placebo stimuli: with a $90^{\circ}$ flipped coil (SHAM), prefrontal (FRO), and peripheral (PERI) stimulation. The latter two were expected to control for two types of aversiveness: the stimulation of the scalp and adjacent face muscles and the peripheral movement of the hand. Therefore, performance effects of frontal and peripheral stimuli were pooled for the ANOVA. Peripheral stimulation above the forearm contralateral to the movement was administered with an intensity that elicited about the same motor response as stimulation over M1 (average intensity of stimulator output: patients, $42.8 \pm 4.2 \%$; HCs, $43.8 \pm 4.4 \%$ ).

Psychological ratings. The rating was obtained with visual analog scales [length of $10 \mathrm{~cm} ; 0$ (no effect) to 10 (maximally aversive)] for both aversiveness and amount of subjective TMS-induced disturbance during the finger sequence. Both ratings were compared for all stimulus locations with a two-way repeated-measures ANOVA with the within-factor location (M1, dPMC, FRO, SHAM, PERI) and the between-factor group (patients, HCs), followed by post hoc $t$ tests corrected for multiple comparisons.

Motor task. Before TMS measurement, both groups trained the same sequence (presented visually) as during the fMRI session. To be able to detect timing errors with high sensitivity during TMS, subjects were instructed to tap precisely at a movement rate of $1 \mathrm{~Hz}$, paced with beep tones. Data were recorded with Presentation (version 0.52; Neurobehavioral Systems, Albany, CA).

Each of the TMS locations was stimulated in a balanced order with 10 runs of a finger sequence for each position before moving on to the next stimulation site. During each finger sequence, a triple pulse with an intensity of $120 \%$ motor threshold and an interpulse interval of $50 \mathrm{~ms}$ (high-frequency jamming with $20 \mathrm{~Hz}$ ) was administered at the accurate time of the $1 \mathrm{~Hz}$ pace for each stimulus location. Additionally, during each of the 10 trials, pulses were administered at a randomized time point between the third and the eighth finger tap. The first two sequences were not used for further evaluation to exclude contamination by novelty of stimulation because of the new location.

Evaluation of performance. The dependent variables of this study were the temporal preciseness of button presses (in milliseconds) and the number of accuracy errors performed between baseline (three taps before the stimulus was applied) and right after TMS pulses (one tap). Differences to normal distribution of the temporal preciseness data were excluded by Kolmogorov-Smirnov tests for each region and time. Statistical evaluation of the temporal preciseness was performed with a threeway repeated-measures ANOVA with the within-factor location [dPMC, M1, SPL, SHAM-control and more aversive-control stimuli (average of PERI and FRO)], stimulation (baseline and post-TMS), and the between-factor group (patients, HCs), followed by post hoc $t$ tests corrected for multiple comparisons.

The accuracy data were not normally distributed and therefore compared with nonparametric tests for independent samples (Mann-Whitney $U$ test; corrected for multiple comparisons). All statistical comparisons were performed with the Statistical Package for the Social Sciences (version 10.05; SPSS, Chicago, IL).

Correlation of blood oxygenation level-dependent effect size and TMS effect. The effect size of the fMRI signal (SPM-betas) in the APMC, M1, and SPL [regions in the normalized data selected after Tzourio-Mazoyer et al. (2002)] was correlated with the TMS effect on temporal preciseness (performance after TMS minus baseline). Pearson correlations were corrected for multiple comparisons.

Relationship of the disruptive effects of TMS applied to the CON-H and the DAM-H. To directly compare the CON-H with the DAM-H in their relevance for recovery of function after brain lesion, all patients were scheduled for a second control experiment. One patient could not join this experiment because of recent surgery; six patients (five men; age, $63.2 \pm 9.3$ years; no changes in functional motor scores between measures) were studied. All patients gave their written informed consent, and the control experiment was approved by the local ethics committee of the 


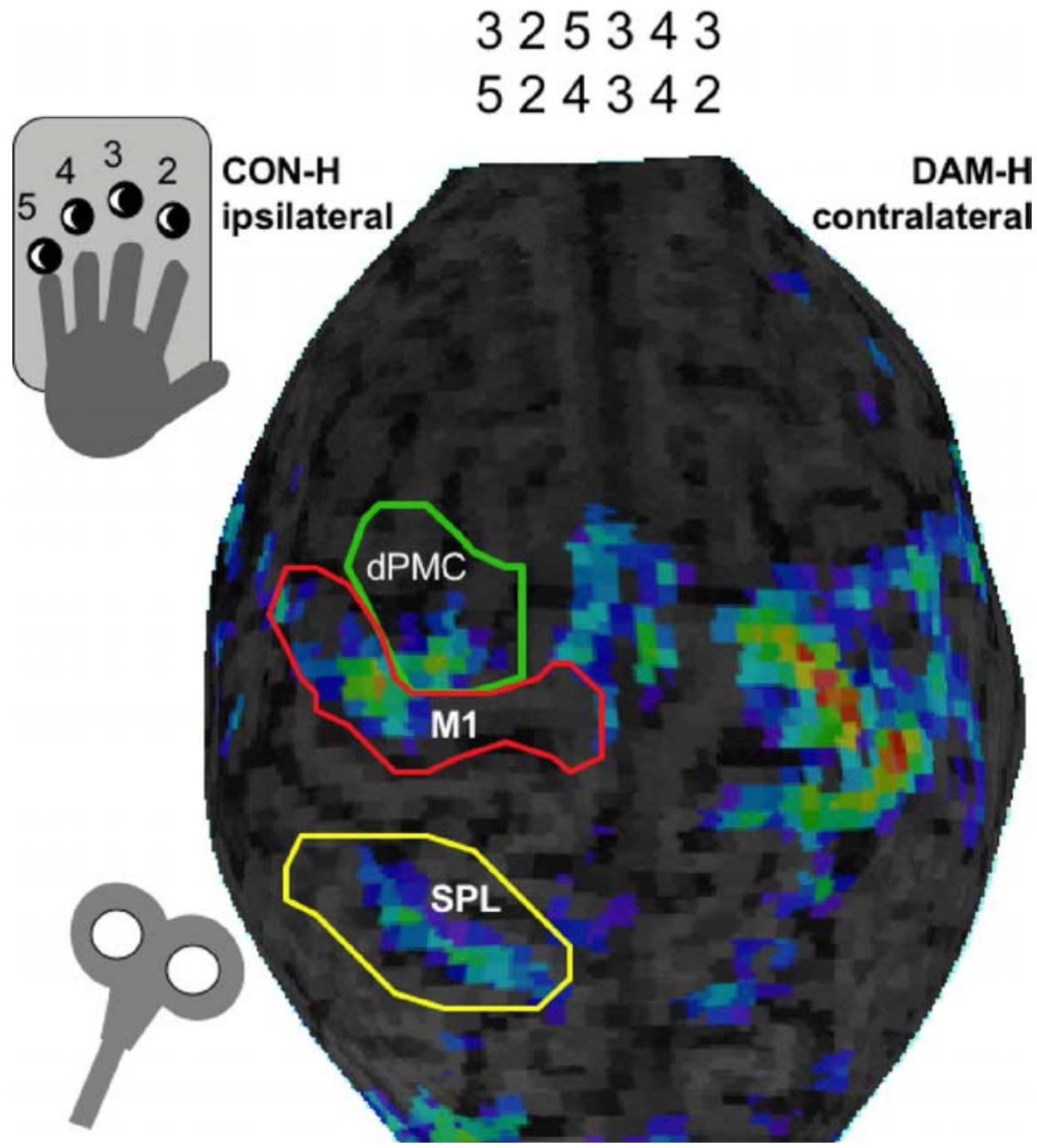

Figure 2. Setting during the experimental paradigm during the TMS experiment. Navigation within different functional representation sites demonstrated in one subject during complex finger sequence performance coded by numbers is shown.

spread out within ROIs (see supplemental Fig. 1, available at www.jneurosci.org as supplemental material). Direct comparison in patients revealed no significant differences between the $1 \mathrm{~Hz}$ and the maximal velocity even when the significance threshold was lowered to $p<0.001$ uncorrected.

\section{Psychological rating during TMS}

During TMS, subjective rating did not differ between patients and HCs at any stimulus location (aversiveness: $F_{(1,12)}=3.25$, NS; disruption of sequences: $F_{(1,12)}=$ 0.059 , NS). There was no interaction of group by location but a significant main effect for location with both types of ratings (aversiveness: $F_{(5,78)}=6.70, p<$ 0.001; disruption: $\left.F_{(5,78)}=2.85, p<0.05\right)$. This indicates that TMS was perceived differently depending on the site of stimulation but that this effect was similar in both groups. Although stimulation over M1, dPMC, SPL, and PERI exerted comparable psychological effects, these conditions differed compared with SHAM stimulation $\left(t_{(13)}>2.57 ; p<0.05\right)$. TMS over FRO stimulation was rated more aversive than the other control conditions (PERI: $t_{(13)}=$ 3.07, $p<0.05$; SHAM: $t_{(13)}=5.86, p<$ $0.001)$. SHAM stimulation was rated less disruptive than $\mathrm{APMC}$ and FRO stimulation $\left(t_{(13)}<2.74 ; p<0.05\right)$ (Fig. 3).

\section{TMS effect on performance}

The resting motor thresholds did not differ between patients (CON-H) and $\mathrm{HCs}$ (average output: patients, $54.1 \pm 6.7 \%$;

Medical Faculty of the University of Tuebingen. Resting motor thresholds of the hand flexors for the recovered and the intact hand were measured. Stimulation intensity for jamming was $120 \%$ of the respective motor threshold. There were no differences between hemispheres (CON-H, 61.3\%; DAM-H, 62.0\%). Neuronavigation was applied with the same technique used in the first experiment. Targets of stimulation were M1, the APMC, and the SPL of both the CON-H and the DAM-H in a randomized order.

\section{Results}

\section{Motor performance and functional maps}

During baseline, no significant differences were seen between patients and HCs in velocity of tapping $\left(t_{(12)}<0.70\right.$; NS) and in error rate of button hits (Mann-Whitney $U$ test; $z<1.5$; NS). This points to an excellent recovery of patients in relation to the performance tested. All fMRI maps of patients and HCs showed activation ipsilateral to the moving hand in the ROIs (Fig. $1 \mathrm{~B}$; see patient group data in supplemental Table 2, available at www. jneurosci.org as supplemental material). On direct comparison, activation ipsilateral to the moving hand (contralesional in patients) was greater in patients than in HCs (Table 1). Scalp localization of the maximum of TMS stimuli over the dPMC was, on average, $30.7 \pm 12.6 \mathrm{~mm}$ more anterolateral to those over M1. One hertz finger sequences revealed activation maxima that primarily matched the topographic pattern observed during movements at maximal frequency, although activated voxels were less
HC, $57.9 \pm 5.8 \%)$. TMS interfered with the temporal precision of the finger sequences. The ANOVA revealed a significant main effect for location $\left(F_{(4,48)}=2.65 ; p<0.05\right)$, stimulation $\left(F_{(1,12)}=\right.$ $49.2 ; p<0.001)$, and the interaction group by stimulation $\left(F_{(1,12)}\right.$ $=5.69 ; p<0.05)$, indicating that TMS interference had a differential effect on patients and HCs. Compared with baseline, timing errors were induced by TMS over M1 $\left(t_{(12)}=2.57 ; p<0.05\right)$, the dPMC $\left(t_{(12)}=3.65 ; p<0.001\right)$, and the SPL $\left(t_{(12)}=2.31 ; p<\right.$ $0.05)$ only for the stroke patients but not for HCs. The differential effect between patients and HCs is illustrated in Figure 4.

Only after SPL stimulation, the number of accuracy errors was larger in patients than in HCs (Mann-Whitney $U$ test; $z=2.67$; $p<0.05)$.

Across all subjects, the fMRI signal intensity within M1 of the $\mathrm{CON}-\mathrm{H}$ correlated positively with the timing errors induced by TMS at this location $(r=0.59 ; p<0.05)$. This correlation was nonsignificant for the dPMC or the SPL, indicating different characteristics of interference at these locations.

In the control experiment, the timing errors elicited by stimulation of the CON-H were comparable to those in the same patients during the original experiment for all regions tested $\left(F_{(1,10)}=0.003\right.$; NS $)$.

The timing errors elicited over the CON-H showed effects comparable to those elicited over the DAM-H in all regions tested $\left(F_{(1,10)}=2.06\right.$; NS). Accuracy errors did not show statistical dif- 


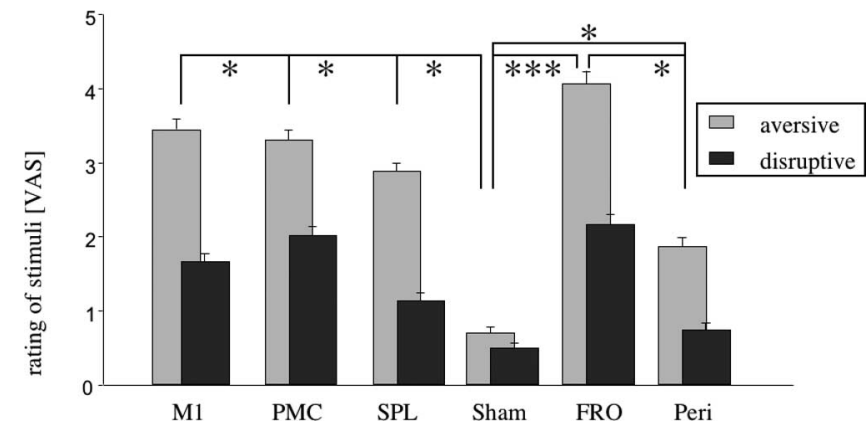

Figure 3. Rating of aversiveness (light gray) and disruptiveness (dark gray) of the stimuli. All stimuli were more aversive and disruptive than SHAM stimuli. Within the control stimuli, the FRO stimuli were more aversive and disruptive than all of the PERI stimuli. Other comparisons did not show significant differences. VAS, Visual analog scale. ${ }^{*} p<0.05$; ${ }^{* * *} p<0.001$.

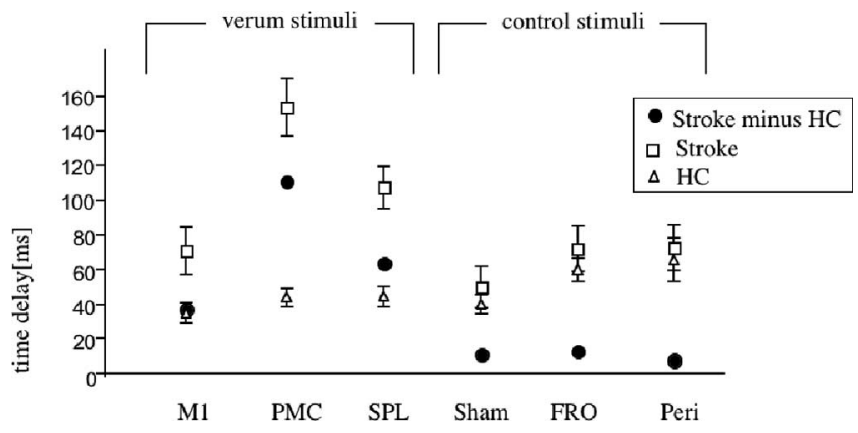

Figure 4. Differences in performance on temporal accuracy; delay from $1000 \mathrm{~ms}(=0)$ after TMS jamming compared with baseline for patients (stroke) and HCs and patients minus HCs. All "verum stimuli" over the CON-H (M1, PMC, and SPL) showed a significant effect selectively for the stroke patients. Control stimuli (SHAM, $90^{\circ}$ upright coil; FRO and PERI over lower arm flexors) showed no differential effect between subject groups.

ferences neither between the original and the control experiment nor between the CON-H and DAM-H. No single pairwise post hoc comparison between the original and control experiment as well as between contralesional and damaged reached significance $\left(t_{(5)}<1\right.$; NS).

In conclusion, only the recovered skilled motor performance in patients was significantly disrupted by TMS over the ipsilateral hemisphere (CON-H). Two patterns of disturbances were observed: (1) timing errors with stimulation over the APMC and M1 and (2) combined timing and accuracy errors with stimulation over SPL. The effect sizes for TMS interference over the CON-H were similar to those over the DAM-H.

\section{Discussion}

In line with previous studies (Weiller et al., 1992; Carey et al., 2002; Ward et al., 2003), the present study showed increased activation in the DAM-H and CON-H of stroke patients. Previous fMRI studies suggested that activation in the CON-H during movements of the affected hand is high in the subacute stage after stroke, decreases over time (Marshall et al., 2000), and lasts to some extent for years after stroke (Carey et al., 2002; Gerloff et al., 2006). The functional relevance of this persistently enhanced activity in the CON-H has been unclear; even negative consequences have been described previously (Ward et al., 2003; Ward, 2004). In fact, restitution of near-normal circuitry is probably the best basis for excellent recovery (Baron et al., 2004; Cramer, 2004). For these patients, also the notion that activity in the $\mathrm{CON}-\mathrm{H}$ increases temporarily after stroke but returns to baseline in the course of effective recovery (Ward et al., 2003) is likely true.
However, in many patients, purely perilesional or ipsilesional reorganization might not be sufficient, and task-related increases of activity in the CON-H persist in the chronic stage, like in our patients.

We used a slightly different task for fMRI than TMS interference: for eliciting significant ipsilateral activation in all ROIs also in HCs, fMRI maps for navigation were generated during the maximal velocity task. These maps did not differ significantly from those generated during $1 \mathrm{~Hz}$ performance, and activation maxima were quite constant for both tasks. It cannot be ruled out that the rhythm keeping in the TMS experiment involved additional cognitive processing. However, because the design was identical for patients and age-matched controls and because the fMRI solely served for adjusting the neuronavigation system, this aspect does not interfere with our main conclusions.

According to recent data, altered interhemispheric interactions after unilateral damage have to be considered. In healthy subjects, motor cortical excitability in both hemispheres is balanced, and, for instance, a decrease in excitability within one hemisphere results in an increase in the other (Plewnia et al., 2003). Because of an imbalance of interhemispheric interactions, a dominance of the CON-H could potentially suppress recovery processes in the DAM-H (Murase et al., 2004). Enhancing excitability of M1 in the DAM-H of stroke patients improves motor performance (Hummel et al., 2005). Thus, upregulated activity in the CON-H might conceivably have adverse effects in some patients. Our data do not argue against that but show that enhanced activation in the CON-H cannot only be seen as an adverse phenomenon.

Transient jamming of cortical activity by TMS allows for conclusions about the functional relevance of regional neuronal activation for a given behavioral task (Cohen et al., 1997). The focal specificity of this method depends on the use of adequate control conditions. Of particular importance is controlling for attentional effects possibly caused by noise or cutaneous sensations inflicted by TMS. The impact of noise was controlled by SHAM stimulation, cutaneous sensations were controlled by FRO stimulation, and possible distraction attributable to movement effects of the nonperforming hand was controlled by PERI stimulation. There were no differences between patients and healthy subjects for these control conditions. Thus, the effects of TMS to the $\mathrm{CON}-\mathrm{H}$ cannot be explained by distraction of attention or a reduced capability of patients to concentrate on the task.

Specific behavioral effects in the patient group were observed for the APMC, SPL, and M1 of the CON-H. Several characteristics qualify these regions as prime candidates for compensatory reorganization and enhanced activity to control recovered motor functions. As the homolog of M1 that exerts corticospinal control under normal conditions, after motor stroke, contralesional M1 is subject to transcallosal disinhibition and shows enhanced excitability (Liepert et al., 2000; Shimizu et al., 2002). Given the close linkage between excitability and plasticity (Butefisch et al., 2000, 2002), this makes the contralesional M1 a reasonable target for corticospinal reorganization (Fisher, 1992). The dPMC and SPL serve as multimodal integration areas, are typically involved in the coding of complex hand movements (Wexler et al. 1997; Catalan et al., 1998), are well interconnected with other frontal and parietal motor-related regions, and are thus well suited to take over functions after lesions of other components of the motor cortical network or its efferent path.

By which mechanism could TMS interference with a single region of the reorganized network affect motor performance? One possibility is that the $\mathrm{APMC}, \mathrm{M} 1$, and SPL of the CON-H are 
subject to higher computational demands after stroke. This would also explain that the predominant role of the SPL in spatiotemporal patterning makes stimulation at this site likely to induce accuracy errors, whereas PMC and M1 stimulation caused timing errors only. After subcortical stroke, the outflow from M1 is compromised and might require more processing in areas outside M1. The lesion of the M1 efferent path may cause an increase in error signals inducing instability in the sensorimotor system. This could be compensated by enhanced processing in other network components such as the $\mathrm{APMC}, \mathrm{M} 1$, and SPL of the CON-H.

The most pronounced effect on timing was seen with stimulation of the dPMC. This is in line with a study using single-pulse TMS to interfere with simple reaction-time movements in stroke patients (Johansen-Berg et al., 2002) and with lesion data indicating that the APMC is important for temporal control of complex movements (Halsband et al., 1993).

Stimulation over the SPL compromised movement timing and accuracy in patients. The SPL, comparable to the dPMC (Freund and Hummelsheim, 1985), is engaged in performance of learned movement sequences (Seitz et al., 1997) and has an additional role for implementing complex sensorimotor tasks (e.g., movement selection based on integration of visual and somatosensory information) (Sadato et al., 1996; Sakai et al., 1998). Compared with healthy subjects (Desmurget et al., 1999), in patients the ipsilateral parietal lobe has apparently gained additional functionality for the correct execution of complex finger sequences. The SPL might serve as a multimodal integration area controlling temporal and spatial preciseness of recovered complex finger movements in the CON-H.

Temporal errors were also induced with TMS to M1 in the CON-H. Other studies, investigating simpler motor paradigms or stroke patients with less complete recovery, did not observe any prominent functional contribution of this area (Foltys et al., 2003; Werhahn et al., 2003). In contrast, previous imaging studies had pointed to $\mathrm{M} 1$ activation of the $\mathrm{CON}-\mathrm{H}$ in stroke patients (Weiller et al., 1992; Carey et al., 2002; Ward et al., 2003). Three methodological aspects of our study are likely the reason for the seeming discrepancies with previous TMS studies. First, we selected the M1 stimulation site in the CON-H strictly based on fMRI-guided neuronavigation. Ipsilateral representation sites in M1 are thought to be more lateral and anterior than the contralateral ones (Wassermann et al., 1994; Ziemann et al., 1999), and therefore stimulation over optimal points for the contalateral hand is not necessarily the most sensitive approach to test contributions to the ipsilateral hand. Second, we selected a complex sequential motor task, thereby maximizing ipsilateral M1 activation. Ipsilateral repetitive TMS of M1 affects complex finger movements, whereas simple movements are not disrupted (Chen et al., 1997). Third, we used a more interfering TMS regimen with multiple instead of single pulses (Johansen-Berg et al., 2002; Werhahn et al., 2003; Fridmann et al., 2004). Together, these points are likely to enhance the sensitivity to detect interferences with recovered behavior in stroke patients.

Of note, the intensity of the fMRI signal in M1 (CON-H) varied across patients and was positively correlated with the effect of jamming over $\mathrm{M} 1$ on temporal preciseness of finger sequences. For the APMC, no such correlation was observed. All patients showed similar increases in fMRI activation in this area. This suggests (1) that the nature and extent of M1 and DPMC contribution to recovered function differ and (2) that the M1 effects are unlikely to be caused by spread of the induced magnetic field from M1 to the APMC. In the latter case, one would expect sim- ilar behavior of both points in the correlation analysis. Field spread is also unlikely because M1 and DPMC stimulation sites were $3.1 \pm 1.3 \mathrm{~cm}$ apart.

It is unlikely that $\mathrm{M} 1$ of the CON-H contributes to motor recovery in capsular stroke through an ipsilateral (uncrossed) monosynaptic corticospinal pathway (Foltys et al., 2003; Gerloff et al., 2006). We favor the interpretation that this area is involved in higher-order motor processing.

Could it simply be that "recovered" movements are relatively more complex for stroke patients and that this explains our data? This view could explain several neuroimaging and neurophysiological results related to recovery after stroke, but it cannot explain that jamming over the SPL resulted in an increase in accuracy errors but not over the $\mathrm{APMC}$ where only timing errors were observed. Therefore, a more specific contribution of areas of the $\mathrm{CON}-\mathrm{H}$ for recovered hand function can be assumed.

Timing errors induced by TMS to the CON-H were comparable to those induced by stimulation of the DAM-H within M1, the $\mathrm{APMC}$, and the SPL, indicating that effective motor recovery after stroke makes use of a distributed neuronal network in both hemispheres.

\section{References}

Baron JC, Cohen LG, Cramer SC, Dobkin BH, Johannsen-Berg H, Loubinoux I, Marshall RS, Ward NS (2004) Neuroimaging in stroke recovery: a position paper from the First International Workshop on Neuroimaging and Stroke Recovery. Cerebrovasc Dis 18:260-267.

Butefisch CM, Davis BC, Wise SP, Sawaki L, Kopylev L, Classen J, Cohen LG (2000) Mechanisms of use-dependent plasticity in the human motor cortex. Proc Natl Acad Sci USA 97:3661-3665.

Butefisch CM, Davis BC, Sawaki L, Waldvogel D, Classen J, Kopylev L, Cohen LG (2002) Modulation of use-dependent plasticity by D-amphetamine. Ann Neurol 51:59-68.

Carey JR, Kimberley TJ, Lewis SM, Auerbach EJ, Dorsey L, Rundquist P, Ugurbil K (2002) Analysis of fMRI and finger tracking training in subjects with chronic stroke. Brain 125:773-788.

Catalan MJ, Honda M, Weeks RA, Cohen LG, Hallet M (1998) The functional neuroanatomy of simple and complex sequential finger movements: a PET study. Brain 121:253-264.

Chen R, Gerloff C, Hallett M, Cohen LG (1997) Involvement of the ipsilateral motor cortex in finger movements of different complexities. Ann Neurol 41:247-254.

Chollet F, DiPiero V, Wise RJ, Brooks DJ, Dolan RJ, Frackowiak RS (1991) The functional anatomy of motor recovery after stroke in humans: a study with positron emission tomography. Ann Neurol 29:63-71.

Cohen LG, Celnik P, Pascual-Leone A, Corwell B, Falz L, Dambrosia J, Honda M, Sadato N, Gerloff C, Catalan MD, Hallet M (1997) Functional relevance of cross-modal plasticity in blind humans. Nature 389:180-183.

Cramer SC (2004) Functional imaging in stroke recovery. Stroke 35: 2695-2698.

Daniels L, Worthingham C (1972) Muscle testing: techniques of manual examination, Ed 3. Philadelphia: Saunders.

Desmurget M, Epstein CM, Turner RS, Prablanc C, Alexander GE, Grafton ST (1999) Role of the posterior parietal cortex in updating reaching movements to a visual target. Nat Neurosci 2:563-567.

Duque J, Hummel F, Celnik P, Murase N, Mazzicchio R, Cohen LG (2005) Transcallosal inhibition in chronic subcortical stroke. NeuroImage 28:940-946.

Fisher CM (1992) Concerning the mechanism of recovery in stroke hemiplegia. Can J Neurol Sci 19:57-63.

Foltys H, Krings T, Meister IG, Sparing R, Boroojerdi B, Thron A, Topper R (2003) Motor representation in patients rapidly recovering after stroke: a functional magnetic resonance imaging and transcranial magnetic stimulation study. Clin Neurophysiol 114:2404-2415.

Freund HJ, Hummelsheim H (1985) Lesions of the premotor cortex in man. Brain 108:697-733.

Fridman EA, Hanakawa T, Chung M, Hummel F, Leiguarda RC, Cohen LG (2004) Reorganization of the human ipsilesional premotor cortex after stroke. Brain 27:747-758. 
Fries W, Danek A, Scheidtmann K, Hamburger C (1993) Motor recovery following capsular stroke. Role of descending pathways from multiple motor areas. Brain 116:369-382.

Gerloff C, Corwell B, Chen R, Hallett M, Cohen LG (1998) The role of the human motor cortex in the control of complex and simple finger movement sequences. Brain 121:1695-1709.

Gerloff C, Bushara K, Sailer A, Wassermann E, Chen R. Waldvogel D, Wittenberg GF, Cohen LG, Hallet M (2006) Multimodal imaging of brain reorganization in motor areas of the contralesional hemisphere of well recovered patients after capsular stroke. Brain 129:791-808.

Halsband U, Ito N, Tanji J, Freund HJ (1993) The role of premotor cortex and the supplementary motor area in the temporal control of movement in man. Brain 116:243-266.

Hummel F, Celnik P, Giraux P, Floel A, Wu WH, Gerloff C, Cohen LG (2005) Effects of non-invasive cortical stimulation on skilled motor function in chronic stroke. Brain 128:490-499.

Johansen-Berg H, Rushworth MF, Bogdanovic MD, Kischka U, Wimalaratna S, Matthews PM (2002a) The role of ipsilateral premotor cortex in hand movement after stroke. Proc Natl Acad Sci USA 99:14518-14523.

Johansen-Berg H, Dawes H, Guy C, Smith SM, Wade DT, Matthews PM (2002b) Correlation between motor improvements and altered fMRI activity after rehabilitative therapy. Brain 125:2731-2742.

Kobayashi M, Hutchinson S, Theoret H, Schlaug G, Pascual-Leone A (2004) Repetitive TMS of the motor cortex improves ipsilateral sequential simple finger movements. Neurology 62:91-98.

Kotila M, Waltimo O, Niemi ML, Laaksonen R, Lempinen M (1984) The profile of recovery from stroke and factors influencing outcome. Stroke 15:1039-1044.

Liepert J, Hamzei F, Weiller C (2000) Motor cortex disinhibition of the unaffected hemisphere after acute stroke. Muscle Nerve 23:1761-1763.

Lyden PD, Zweifler R, Mahdavi Z, Lonzo L (1994) A rapid, reliable, and valid method for measuring infarct and brain compartment volumes from computed tomographic scans. Stroke 25:2421-2428.

Maier MA, Armand J, Kirkwood PA, Yang HW, Davis JN, Lemon RN (2002) Differences in the corticospinal projection from primary motor cortex and supplementary motor area to macaque upper limb motoneurons: an anatomical and electrophysiological study. Cereb Cortex 12:281-296.

Marshall RS, Perera GM, Lazar RM, Krakauer JW, Constantine RC, DeLaPaz RL (2000) Evolution of cortical activation during recovery from corticospinal tract infarction. Stroke 31:656-661.

Murase N, Duque J, Mazzocchio R, Cohen LG (2004) Influence of interhemispheric interactions on motor function in chronic stroke. Ann Neurol 55:400-409.

Nudo RJ, Wise BM, SiFuentes F, Milliken GW (1996) Neural substrates for the effects of rehabilitative training on motor recovery after ischemic infarct. Science 272:1791-1794.

Oldfield RC (1971) The assessment and analysis of handedness: the Edinburgh inventory. Neuropsychologia 9:97-113.

Plewnia C, Lotze M, Gerloff C (2003) Disinhibition of the contralateral motor cortex by low-frequency rTMS. NeuroReport 14:609-612.
Rossini PM, Narici L, Martino G, Pasquarelli A, Peresson M, Pizzella V, Tecchio F, Romani GL (1994) Analysis of interhemispheric asymmetries of somatosensory evoked magnetic fields to right and left median nerve stimulation. Electroencephal Clin Neurophysiol 91:476-482.

Sadato N, Campbell G, Ibanez V, Deiber M, Hallett M (1996) Complexity affects regional cerebral blood flow change during sequential finger movements. J Neurosci 16:2691-2700.

Sakai K, Hikosaka O, Miyauchi S, Takino R, Sasaki Y, Putz B (1998) Transition of brain activation from frontal to parietal areas in visuomotor sequence learning. J Neurosci 18:1827-1840.

Seitz RJ, Canavan AG, Yaguez L, Herzog H, Tellmann L, Knorr U, Huang Y, Homberg V (1997) Representations of graphomotor trajectories in the human parietal cortex: evidence for controlled processing and automatic performance. Eur J Neurosci 9:378-389.

Seitz RJ, Hoflich P, Binkofski F, Tellmann L, Herzog H, Freund HJ (1998) Role of the premotor cortex in recovery from middle cerebral artery infarction. Arch Neurol 55:1081-1088.

Shimizu T, Hosaki A, Hino T, Sato M, Komori T, Hirai S, Rossini PM (2002) Motor cortical disinhibition in the unaffected hemisphere after unilateral cortical stroke. Brain 125:1896-1907.

Tzourio-Mazoyer N, Landeau B, Papathanassiou D, Crivello F, Etard O, Delcroix N, Mazoyer B, Joliot M (2002) Automated anatomical labeling of activations in SPM using a macroscopic anatomical parcellation of the MNI MRI single-subject brain. NeuroImage 15:273-289.

Ward NS (2004) Functional reorganization of the cerebral motor system after stroke. Curr Opin Neurol 17:725-730.

Ward NS, Brown MM, Thomson AJ, Frackowiak RS (2003) Neural correlates of outcome after stroke: a cross sectional fMRI-study. Brain 126:1430-1448.

Wassermann EM, Pascual-Leone A, Hallett M (1994) Cortical motor representation of the ipsilateral hand and arm. Exp Brain Res 100:121-132.

Weiller C, Chollet F, Friston KJ, Wise RJ, Frackowiak RS (1992) Functional reorganization of the brain in recovery from striatocapsular infarction in man. Ann Neurol 31:463-472.

Werhahn KJ (1994) The effect of magnetic coil orientation on the latency of surface EMG and single motor unit responses in the first dorsal interosseous muscle. Electroencephal Clin Neurophysiol 93:138-146.

Werhahn KJ, Conforto AB, Kadom N, Hallett M, Cohen LG (2003) Contribution of the ipsilateral motor cortex to recovery after chronic stroke. Ann Neurol 54:464-472.

Wexler BE, Fulbright RK, Lacadie CM, Skudlarski P, Kelz MB, Constable RT, Gore JC (1997) An fMRI study of the human cortical motor system response to increasing functional demands. Reson Imaging 15:385-396.

Yousry TA, Schmid UD, Alkadhi H, Schmidt D, Peraud A, Buettner A, Winkler P (1997) Localization of the motor hand area to a knob on the precentral gyrus. A new landmark. Brain 120:141-157.

Ziemann U, Ishii K, Borgheresi A, Yaseen Z, Battaglia F, Hallett M, Cincotta M, Wassermann EM (1999) Dissociation of the pathways mediating ipsilateral and contralateral motor-evoked potentials in human hand and arm muscles. J Physiol (Lond) 518:895-906. 